\title{
A Competing-Risk Nomogram Predicting Second Primary Malignancy in Hepatocellular Carcinoma Survivors
}

\section{Xuqi Sun}

Sun Yat-sen University Cancer Center Jinbin Chen

Sun Yat-sen University Cancer Center Juncheng Wang

Sun Yat-sen University Cancer Center

Zhongguo Zhou

Sun Yat-sen University Cancer Center

Li Xu

Sun Yat-sen University Cancer Center

Yaojun Zhang

Sun Yat-sen University Cancer Center

\section{Dandan Hu}

Sun Yat-sen University Cancer Center

MS Chen ( $D$ chenminshansysucc@163.com )

Sun Yat-sen University Cancer Center

\section{Research article}

Keywords: risk factors, hepatocellular carcinoma, second primary malignancy, nomogram

Posted Date: April 14th, 2020

DOI: https://doi.org/10.21203/rs.3.rs-21764/v1

License: (c) (i) This work is licensed under a Creative Commons Attribution 4.0 International License. Read Full License 


\section{Abstract}

Background:Hepatocellular carcinoma (HCC) survivors are increasing due to the improvement of survival. Follow-up scheme to prevent recurrence is routinely recommended, but are these patients exposed to increased risks of second malignancies remains unknown. We aimed to identify risk factors for second primary malignancies (SPM) among HCC survivors and develop a predicting model accordingly.

Methods: We identified HCC patients in the Surveillance, Epidemiology, and End Results (SEER) database from 2004 to 2014. For the incidence of specific SPMs, standardized incidence ratios (SIRs) were calculated. The proportional hazard model was performed in order to discover hazards of SPM, and a competing-risk nomogram was developed to stratify patients with different incidences for SPM.

Results: The crude incidence of SPM was $2.70 \%$ in HCC survivors, with the SIR of 1.09 . HCC survivors had significantly higher incidences of SPM in the following sites: oral cavity and pharynx, stomach, intra and extrahepatic bile duct, lung and bronchus, kidney and renal pelvis, thyroid and lymphatic system $(\mathrm{p}<$ 0.05). Patients with diagnosed age at 61-75 years old, smaller tumor size, better histologic differentiation, earlier AJCC stage or surgical history had higher risks for SPM. The c-index of the competing-risk nomogram was 0.663 . The cumulative incidences of SPM were significantly different among each quartile of patients stratified by the predicting model $(p<0.05)$.

Conclusion:HCC survivors had an excess risk for SPMs, especially those with promising prognostic factors. This nomogram was the first competing-risk model for identifying high-risk cohort and assist clinical screening.

\section{Introduction}

Liver cancer ranks as the sixth most common malignancy, and the number of new cases is approximately 800,000 per year [1]. Over $90 \%$ of liver cancer cases are hepatocellular carcinoma (HCC), the incidence of which has been rising over the last two decades [2]. The survival of HCC has improved due to early diagnosis of disease as well as better surveillance and treatment modalities [3]. With the improvement of prognosis, studies on the management of HCC survivors are urgent.

Theoretically, patients with cancer history have increasing risks for subsequent tumors due to genetic mutations, hazard environmental exposure and unhealthy lifestyles $[4,5]$. Previous studies reported that patients with colorectal cancer, lymphoma and thyroid cancer have higher incidences of second primary malignancy (SPM) [6-8]. However, such studies regarding HCC are limited. Heo $\mathrm{J}$ et al reported that the incidence of SPM was $4.2 \%$ among HCC patients after liver transplantation [9]. While one research from Taiwan showed a standardized incidence ratio (SIR) of 0.88 for SPM in the whole HCC patients, another study reported HCC patients had higher incidences for SPM compared with the standardized population $[10,11]$. Mario et al reported that $18(7.3 \%)$ HCC patients had synchronous or metachronous extrahepatic malignancy [12]. The above studies were performed quite a few years ago, when treatments like ablation or sorafenib for HCC were not commonly applied. The improved survival for HCC currently may cause a 
higher incidence of SPM than older studies. Besides, previous studies were mostly observational research and did not demonstrate specific risk factors for SPM. Given the increasing number of HCC survivors, identifying and surveilling high-risk population for SPM is urgent.

We aimed to evaluate the incidence of SPM in HCC survivors in this study, to identify the risk factors for subsequent cancers and to develop a competing-risk nomogram for assisting surveillance in HCC patients.

\section{Methods}

\section{Study Population}

We identified patients diagnosed with primary HCC in the Surveillance, Epidemiology, and End Results (SEER) database between 2004 and 2014. Patients were excluded with one of the following conditions: 1) with only autopsy or death certification, 2) age at diagnosis < 18 years old, 3) without active follow-up. SPM was defined as a new primary malignancy diagnosed at least 3 months after the first primary cancer [13]. In this study, patients with the same SPM as primary ones were excluded since HCC is prone to recurrence [14]. Data of demographic and clinical characteristics were recorded.

\section{Statistical Analysis}

The number of SPM cases was divided by that of the whole patients for the crude incidence of SPM. SIR was the ratio of the incidence of SPM in HCC survivors to the expected incidence in general population, which was calculated by the multiple primary SIR set of SEER*Stat. Chi-squared tests were adopted to evaluate how demographic and clinical factors affected the incidence of SPM in terms of age at diagnosis, race, gender, marital status, histologic grade, tumor size, AJCC stage and surgical history. With the consideration of competing risk, a proportional sub-distribution hazard model was adopted for estimating the hazard of SPM so as to identify the association of covariates and SPM incidences. In the competing-risk analysis, SPM and deaths were considered to be competing events. Furthermore, cumulative incidence function (CIF) was adopted for assessing the probability of events, and Gray's test was performed to evaluate the differences of CIF among subgroups. We then develop a competing-risk nomogram to assess the 1-, 3-, 5-, and 10-year probability of SPM in HCC survivors. The performance of nomogram was assessed by discrimination and calibration with 100 bootstrap resampling.

Discrimination was evaluated by the index of concordance probability (c-index). Calibration was presented with calibration curves. Considering the deficiency of c-index, we also evaluated the classifying ability of nomogram by dividing patients into quartiles based on estimated risk scores [15]. All the statistical analyses were progressed by $\mathrm{R}$ version 3.5 .3 software. Significant difference was set at $P$ value less than 0.05 .

\section{Results}

\section{Incidences of SPM after HCC}


Among 43556 patients diagnosed with HCC, 1176 suffered from SPM. The crude incidence of SPM was $2.70 \%$. Patients with SPM were younger at the diagnosis of HCC than those without (62.41 vs 62.91 years old, $p<0.001$ ) (Table 1). No significant difference was found in the distribution of gender and race between patients with and without SPM ( $p=0.584$ and 0.555$)$. The incidence of SPM increased with age at $61-75$ years old $(3.18 \%)$, others/unknown race (2.84\%), married (3.01\%), tumor size $\leq 3 \mathrm{~cm}(3.60 \%)$, well or moderately differentiated (I-II, 3.79\%), early AJCC stage $(\mathrm{I}, 3.35 \%)$ and surgical history $(5.01 \%)$. The cumulative incidence curves are presented in Fig. 1. 
Table 1

Crude incidence of Second Primary Malignancy in HCC patients

\begin{tabular}{|c|c|c|c|c|}
\hline Characteristics & All patients $(n)$ & SPM patients (n) & SPM crude incidence (\%) & $P$ value \\
\hline Total & 43556 & 1176 & 2.70 & \\
\hline Age (years) & & & & $<0.001$ \\
\hline$<60$ & 20570 & 503 & 2.44 & \\
\hline $61-75$ & 16748 & 532 & 3.18 & \\
\hline$>75$ & 6238 & 141 & 2.26 & \\
\hline Sex & & & & 0.607 \\
\hline Male & 32668 & 874 & 2.68 & \\
\hline Female & 10888 & 302 & 2.77 & \\
\hline Race & & & & 0.555 \\
\hline White & 29523 & 793 & 2.69 & \\
\hline Black & 5417 & 138 & 2.55 & \\
\hline Others/unknown & 8616 & 245 & 2.84 & \\
\hline Marital status & & & & $<0.001$ \\
\hline Unmarried & 20561 & 485 & 2.36 & \\
\hline Married & 22995 & 691 & 3.01 & \\
\hline Size $(\mathrm{cm})$ & & & & $<0.001$ \\
\hline$\leq 3$ & 11084 & 399 & 3.60 & \\
\hline $3-5$ & 8658 & 243 & 2.81 & \\
\hline $5-10$ & 9052 & 178 & 1.97 & \\
\hline$>10$ & 3992 & 60 & 1.50 & \\
\hline Unknown & 10770 & 296 & 2.75 & \\
\hline Histologic grade & & & & $<0.001$ \\
\hline $\mid-I I$ & 12906 & 489 & 3.79 & \\
\hline III-IV & 3705 & 89 & 2.40 & \\
\hline Unknown & 26945 & 598 & 2.22 & \\
\hline
\end{tabular}

SPM, second primary malignancy. 


\begin{tabular}{|lllll|}
\hline Characteristics & All patients $(\mathbf{n})$ & SPM patients (n) & SPM crude incidence (\%) & P value \\
\hline AJCC stage & & & & $<0.001$ \\
\hline I & 14077 & 472 & 3.35 & \\
\hline II & 7640 & 250 & 3.27 & \\
\hline III & 7371 & 110 & 1.49 & $<0.001$ \\
\hline IV & 4192 & 31 & 0.74 & \\
\hline Unknown & 10276 & 313 & 3.05 & \\
\hline Surgical history & & & & \\
\hline No & 29808 & 487 & 1.63 & \\
\hline Yes & 13748 & 689 & 5.01 & \\
\hline SPM, second primary malignancy. & & & \\
\hline
\end{tabular}

\section{SIRs of Specific Cancers after HCC}

The SIRs of specific cancers after HCC are shown in Table 2. The SIR of SPM was 1.09 for the whole HCC patients. The most common systems with SPM were respiratory $(21.1 \%)$, digestive $(18.7 \%)$ and urinary systems (9.3\%). Increased SIRs of cancer were found in the following sites: oral cavity and pharynx, stomach, intra and extrahepatic bile duct, lung and bronchus, kidney and renal pelvis, thyroid and lymphatic system. Decreased SIRs of cancer were found in the following sites: colon and rectum, breast and prostate. 
Table 2

Standardized Incidence Ratio of Second Primary Malignancies in Patients with HCC

\begin{tabular}{|c|c|c|c|}
\hline Site of Malignancies & Observed & Expected & $\operatorname{SIR}(95 \% \mathrm{CI})$ \\
\hline All sites & 1176 & 1075.11 & $1.09(1.03-1.16)$ \\
\hline Oral cavity and pharynx & 59 & 32.14 & $1.84(1.40-2.37)$ \\
\hline Digestive system & 220 & 199.45 & $1.10(0.96-1.26)$ \\
\hline Stomach & 47 & 23.07 & $2.04(1.50-2.71)$ \\
\hline Colon and rectum & 74 & 107.38 & $0.69(0.51-0.90)$ \\
\hline Intra and extrahep bile duct & 30 & 7.86 & $3.82(2.58-5.45)$ \\
\hline Pancreas & 37 & 32.55 & $1.14(0.80-1.57)$ \\
\hline Respiratory system & 248 & 170.67 & $1.45(1.28-1.65)$ \\
\hline Lung and bronchus & 232 & 157.79 & $1.47(1.29-1.67)$ \\
\hline Soft tissue including heart & 8 & 6.31 & $1.27(0.55-2.50)$ \\
\hline Skin excluding basal and squamous & 42 & 49.60 & $0.85(0.61-1.14)$ \\
\hline Breast & 51 & 72.44 & $0.70(0.52-0.93)$ \\
\hline Female breast & 50 & 70.53 & $0.71(0.53-0.93)$ \\
\hline Female genital system & 30 & 27.60 & $1.09(0.73-1.55)$ \\
\hline Male genital system & 143 & 264.07 & $0.54(0.46-0.64)$ \\
\hline Prostate & 141 & 260.75 & $0.54(0.46-0.64)$ \\
\hline Urinary system & 109 & 103.80 & $1.05(0.86-1.27)$ \\
\hline Urinary bladder & 52 & 60.17 & $0.86(0.65-1.13)$ \\
\hline Kidney and renal pelvis & 57 & 41.12 & $1.39(1.05-1.80)$ \\
\hline Brain & 7 & 11.51 & $0.61(0.24-1.25)$ \\
\hline Endocrine system & 37 & 16.23 & $2.28(1.60-3.14)$ \\
\hline Thyroid & 34 & 14.91 & $2.28(1.58-3.19)$ \\
\hline Lymphoma & 119 & 48.55 & $2.45(2.03-2.93)$ \\
\hline Myeloma & 15 & 17.05 & $0.88(0.49-1.45)$ \\
\hline Leukemia & 29 & 28.79 & $1.01(0.67-1.45)$ \\
\hline
\end{tabular}




\section{Risk Factors for SPM after HCC}

After proportional sub-distribution hazard analysis, age at diagnosis of HCC, tumor size, histologic grade, AJCC stage and surgical history had significant correlation with the risk of SPM (Table 3). Patients diagnosed with HCC at 61-75 years old had higher risk of SPM with a sub-distributional hazard ratio (HR) of 1.44 (95\% Cl:1.27-1.63). The risk of SPM increased as the tumor size decreased. Better differentiated tumor indicated higher risk of SPM. Patients with earlier AJCC stage were more likely to develop SPM. Those receiving surgery for initial HCC had significant higher risk for SPM, with a HR of 2.48 (95\% Cl: 2.17-2.83). 
Table 3

Proportional Sub-distribution Hazards Model for the Incidence of SPM

\begin{tabular}{|c|c|c|c|}
\hline Characteristics & Coefficient & $\mathrm{HR}(95 \% \mathrm{Cl})$ & $P$ value \\
\hline \multicolumn{4}{|l|}{ Age (years) } \\
\hline$<60$ & reference & & \\
\hline $61-75$ & 0.362 & $1.436(1.269-1.625)$ & $<0.001$ \\
\hline$>75$ & 0.176 & $1.193(0.980-1.452)$ & 0.078 \\
\hline \multicolumn{4}{|l|}{ Sex } \\
\hline Male & reference & & \\
\hline Female & -0.021 & $0.979(0.855-1.121)$ & 0.760 \\
\hline \multicolumn{4}{|l|}{ Race } \\
\hline White & reference & & \\
\hline Black & 0.069 & $1.071(0.891-1.289)$ & 0.460 \\
\hline Others/unknown & -0.029 & $0.972(0.840-1.124)$ & 0.700 \\
\hline \multicolumn{4}{|l|}{ Marital status } \\
\hline Unmarried & reference & & \\
\hline Married & 0.097 & $1.102(0.976-1.244)$ & 0.120 \\
\hline \multicolumn{4}{|l|}{ Size $(\mathrm{cm})$} \\
\hline$\leq 3$ & reference & & \\
\hline $3-5$ & -0.169 & $0.844(0.719-0.992)$ & 0.040 \\
\hline $5-10$ & -0.262 & $0.770(0.631-0.940)$ & 0.010 \\
\hline$>10$ & -0.494 & $0.610(0.457-0.814)$ & 0.001 \\
\hline Unknown & -0.238 & $0.788(0.633-0.982)$ & 0.034 \\
\hline \multicolumn{4}{|l|}{ Histologic grade } \\
\hline I-II & reference & & \\
\hline III-IV & -0.259 & $0.771(0.615-0.967)$ & 0.025 \\
\hline Unknown & -0.128 & $0.880(0.774-1.000)$ & 0.050 \\
\hline
\end{tabular}

$\mathrm{HR}$, hazard ratio; $\mathrm{Cl}$, confidence interval; SPM, second primary malignancy. 


\begin{tabular}{|llll|}
\hline Characteristics & Coefficient & HR $(95 \%$ Cl) & P value \\
\hline I II & reference & & \\
III & -0.057 & $0.945(0.807-1.107)$ & 0.480 \\
IV & -0.465 & $0.628(0.499-0.791)$ & $<0.001$ \\
Unknown & -1.000 & $0.368(0.251-0.540)$ & $<0.001$ \\
Surgical history & 0.014 & $1.014(0.826-1.246)$ & 0.890 \\
\hline No & & & \\
Yes & reference & & $<0.001$ \\
\hline HR, hazard ratio; Cl, confidence interval; SPM, second primary malignancy. & \\
Oment and EValuation of the Competing-risk
\end{tabular}

Figure 2 presents the competing-risk nomogram for predicting the 1-,3-,5- and 10-year probability of SPM based on Fine -Gray model. By summing up the points of each characteristic, we can predict the incidence of SPM in each year. The c-index of our model was 0.663 , with the area under curve (AUC) being $0.621,0.649,0.666$ and 0.701 in $1,3,5$, and 10 years respectively. The calibration curves are plotted in Fig. 3. To further validate the classifying ability of our model, patients were differentiated into four quartiles based on their points calculated from the nomogram. The cumulative risks of SPM for each quartile were estimated (Fig. 4). The incidences of SPM were significantly different among these groups $(p<0.01)$. Therefore, our nomogram could identify high-risk group for SPM well and assist clinical surveillance.

\section{Discussion}

In this study, we found $2.70 \%(1176 / 43556)$ patients suffered from SPM after initial HCC diagnosis. The SIR of SPM was 1.09 in HCC patients, which indicated that HCC survivors were more likely to develop SPM, comparing with the general population. Furthermore, increased SIRs of cancer were found in the oral cavity and pharynx, stomach, intra and extrahepatic bile duct, lung and bronchus, kidney and renal pelvis, thyroid and lymphatic system Previous studies have reported the incidence of SPM in HCC patients ranged from $1.63-4.2 \%$, and they also found significant higher risks for SPM in the above organs [9-11]. Several reasons can explain the increased incidences of SPM in specific sites. First, impaired liver function caused by cirrhosis can evoke carcinogenesis by distribution of hormones and chemicals, which increases risks for extrahepatic malignancy [16]. In addition, virus infection and tobacco use are common risk factors for liver cancer, lymphoma, respiratory and digestive system cancers $[16,17]$. 
Most of the previous studies reporting SPM after initial HCC were performed more than one decade ago or focused only on patients after liver transplantation $[9-11,18]$. Besides, specific risk factors for SPM were not identified. With the advancements in therapy for $\mathrm{HCC}$ and surveillance for high-risk population, the survival of HCC patients has been prolonged[19]. For instance, ablation was recommended as firstline radical treatment for small HCCs as curative treatment modalities [19]. In 2007, Sorafenib, an oral multitargeted kinase inhibitor, was approved as the first-line treatment for advanced HCC officially [20]. Since then, other targeted therapy and immune checkpoint inhibitors were also applied in clinical practice. Given that cancer is an age-related disease, with the joint efforts mentioned above and the aging of population, the number of survivors with multiple primary malignancies continue to grow. How to improve physical and psychological health of this vulnerable population remains unclear. Developing a predicting model can benefit patients' survival by early detection of subsequent tumors.

A competing-risk nomogram was developed in this study to evaluate risks of SPM in HCC survivors. Our results indicated that patients with HCC diagnosed age at 61-75 years old, smaller tumor size, better histologic differentiation, earlier AJCC stage or surgical history had higher risk of SPM. In summary, patients with promising factors for survival tended to suffer from SPM, which agreed with the results from lung cancer [21]. Our model had good discrimination ability in stratifying risks of SPM. Most previous predicting nomograms were built according to results from Kaplan-Meier or COX proportional hazards methods, which can only deal with single outcome. But for the study of SPM, competing events can have significant impact on results since some patients died of other causes before the diagnosis of a second tumor. To our knowledge, this nomogram is the first competing-risk predicting model for SPM in HCC survivors.

The most common sites of SPMs were lung and bronchus, colon and rectum, prostate, lymph, oral cavity and pharynx, and urinary bladder in this study. In the United States (US), the top five malignancies were lung, colon, prostate, melanoma of the skin and urinary bladder cancers [22]. Though the first three cancers were similar in the general and HCC population, patients with HCC had significantly higher risks of lung and bronchus cancers. In addition, the incidence of lymphoma apparently increased in survivors of HCC, which might attribute to the shared risk factors or the use of anti-virus drugs [23]. These findings can be applied in the management of HCC survivors. We can identify the high-risk patients for SPMs in the first place and lay more emphasis on the screening for those high-risk subsequent tumors. Low-dose helical computer tomography should be strictly performed in HCC survivors with smoking, not only for surveilling recurrence but for detecting lung cancer timely. Lymphoma is noteworthy in HCC patients for more than doubling hazard rations compared with general population. Screening for oral cavity and pharynx cancers such as physical examination and laryngoscopy is considerable during the follow-up in HCC patients. The incidences of colon and prostate cancers were not increased in HCC survivors, but general screening like carcinoembryonic antigen and prostate-specific antigen tests ought to be adopted according to current guidelines due to relatively high morbidities [24, 25].

This study was a population-based research from the SEER database, which includes approximately $30 \%$ of the US population and has unified criteria for data collection. However, there were also several 
limitations. The principle concern was the absence of detailed clinical information such as treatment modalities, reports of imaging, and laboratory tests. Besides, family history, living habits and environmental exposure were also unavailable, which are potential risk factors for subsequent cancers [4, 5]. Another problem is that misdiagnosis of metastasis as SPM might exist in some patients, though we only enrolled patients with cancer interval period longer than three months. Albeit above drawbacks, our predicting model can stratify HCC survivors with different risks of SPM and assist clinical surveillance.

\section{Conclusions}

In conclusion, HCC survivors with better prognosis had increasing risks for subsequent cancers. Clinicians should be aware that increased risks of the below malignancies exist for HCC patients, and make more appropriate follow-up plan accordingly: oral cavity and pharynx, stomach, intra and extrahepatic bile duct, lung and bronchus, kidney and renal pelvis, thyroid and lymphoma. Our competingrisk nomogram can stratify patients with different risks for SPMs.

\section{List Of Abbreviations}

$\mathrm{CIF}$, cumulative incidence function; c-index, index of concordance probability; $\mathrm{HCC}$, hepatocellular carcinoma; SEER, Surveillance, Epidemiology, and End Results; SIR, standardized incidence ratio; SPM, second primary malignancy; US, the United States

\section{Declarations}

Ethical approve and consent to participate

This study was approved by the institutional review board of Sun Yat-sen University Cancer Center as a retrospective study based on the public database, and the requirement for informed consent was waived. All procedures performed in studies involving human participants were in accordance with the ethical standards of the1964 Helsinki declaration and its later amendments.

Consent for publication

None.

Availability of data and material

All data included in this study are available upon request by contact with the corresponding author. Competing interests:

The authors declare that they have no conflict of interest.

Funding:

This study received no funding support.

Authors' contributions:

(I) Study design: M.S., D.H. and X.S.; (II) Administrative support: M.S. and D.H.; (III) Collection and assembly of data: X.S., J.C., L.X. and Y.Z; (IV) Data analysis and interpretation: Z.Z., J.C. ,J.W. and Z.Z.; (V) Manuscript writing: All authors; $(\mathrm{VI})$ Final approval of manuscript: All authors. Acknowledgements: 
None.

\section{References}

1. Bray F, Ferlay J, Soerjomataram I, et al. Global cancer statistics 2018: GLOBOCAN estimates of incidence and mortality worldwide for 36 cancers in 185 countries. CA Cancer J Clin. 2018;68(6):394-424.

2. Petrick JL, Kelly SP, Altekruse SF, et al. Future of Hepatocellular Carcinoma Incidence in the United States Forecast Through 2030. J Clin Oncol. 2016;34(15):1787-94.

3. Kulik L, El-Serag HB. Epidemiology and Management of Hepatocellular Carcinoma. Gastroenterology. 2019;156(2):477-91. e1.

4. Mariotto $A B$, Rowland JH, Ries LA, et al. Multiple cancer prevalence: a growing challenge in long-term survivorship. Cancer Epidemiol Biomarkers Prev. 2007;16(3):566-71.

5. Travis LB, Gilbert E. Lung cancer after Hodgkin lymphoma: the roles of chemotherapy, radiotherapy and tobacco use. Radiat Res. 2005;163(6):695-6.

6. Chattopadhyay S, Sud A, Zheng G, et al. Second primary cancers in non-Hodgkin lymphoma: Bidirectional analyses suggesting role for immune dysfunction. Int J Cancer. 2018;143(10):2449-57.

7. He X, Wu W, Ding Y, et al. Excessive risk of second primary cancers in young-onset colorectal cancer survivors. Cancer Med. 2018;7(4):1201-10.

8. Kim C, Bi X, Pan D, et al. The risk of second cancers after diagnosis of primary thyroid cancer is elevated in thyroid microcarcinomas. Thyroid. 2013;23(5):575-82.

9. Heo J, Noh OK, Oh YT, et al. Second primary cancer after liver transplantation in hepatocellular carcinoma: a nationwide population-based study. Hepatol Int. 2017;11(6):523-8.

10. Shah BK, Kandel P, Khanal A. Second Primary Malignancies in Hepatocellular Cancer - A US Population-based Study. Anticancer Res. 2016;36(7):3511-4.

11. Wu WC, Chen YT, Hwang CY, et al. Second primary cancers in patients with hepatocellular carcinoma: a nationwide cohort study in Taiwan. Liver Int. 2013;33(4):616-23.

12. Fernandez-Ruiz M, Guerra-Vales JM, Castelbon-Fernandez FJ, et al. Multiple primary malignancies in Spanish patients with hepatocellular carcinoma: analysis of a hospital-based tumor registry. $\mathrm{J}$ Gastroenterol Hepatol. 2009;24(8):1424-30.

13. Krueger H, McLean D, Williams D. Meaning of SPC. Prog Exp Tumor Res. 2008;40:7-16.

14. Marrero JA, Kulik LM, Sirlin CB, et al. Diagnosis, Staging, and Management of Hepatocellular Carcinoma: 2018 Practice Guidance by the American Association for the Study of Liver Diseases. Hepatology. 2018;68(2):723-50.

15. Cook NR, Ridker PM. Advances in measuring the effect of individual predictors of cardiovascular risk: the role of reclassification measures. Ann Intern Med. 2009;150(11):795-802.

16. Sorensen HT, Friis S, Olsen JH, et al. Risk of liver and other types of cancer in patients with cirrhosis: a nationwide cohort study in Denmark. Hepatology. 1998;28(4):921-5. 
17. Mahale P, Torres HA, Kramer JR, et al. Hepatitis $C$ virus infection and the risk of cancer among elderly US adults: A registry-based case-control study. Cancer. 2017;123(7):1202-11.

18. Oweira H, Schmidt J, Helbling D, et al. Impact of liver transplantation on the risk of second malignant tumors among hepatocellular carcinoma patients. Expert Rev Gastroenterol Hepatol. 2017;11(9):865-9.

19. Vogel A, Cervantes A, Chau I, et al. Hepatocellular carcinoma: ESMO Clinical Practice Guidelines for diagnosis, treatment and follow-up. Ann Oncol. 2018;29(Suppl 4):iv238-55.

20. Li D, Sedano S, Allen R, et al. Current Treatment Landscape for Advanced Hepatocellular Carcinoma: Patient Outcomes and the Impact on Quality of Life. Cancers (Basel). 2019;11(6).

21. Zhou H, Shen J, Zhang Y, et al. Risk of second primary malignancy after non-small cell lung cancer: a competing risk nomogram based on the SEER database. Ann TransI Med. 2019;7(18):439.

22. Siegel RL, Miller KD, Jemal A. Cancer statistics, 2019. CA Cancer J Clin. 2019;69(1):7-34.

23. Nakamura $Y$, Motokura T, Fujita A, et al. Severe hepatitis related to chemotherapy in hepatitis B virus carriers with hematologic malignancies. Survey in Japan, 1987-1991. Cancer. 1996;78(10):2210-5.

24. Gupta S, Sussman DA, Doubeni CA, et al. Challenges and possible solutions to colorectal cancer screening for the underserved. J Natl Cancer Inst. 2014;106(4):dju032.

25. Ilic D, Djulbegovic M, Jung JH, et al. Prostate cancer screening with prostate-specific antigen (PSA) test: a systematic review and meta-analysis. BMJ. 2018;362:k3519.

\section{Figures}


Age $(P<0.001)$

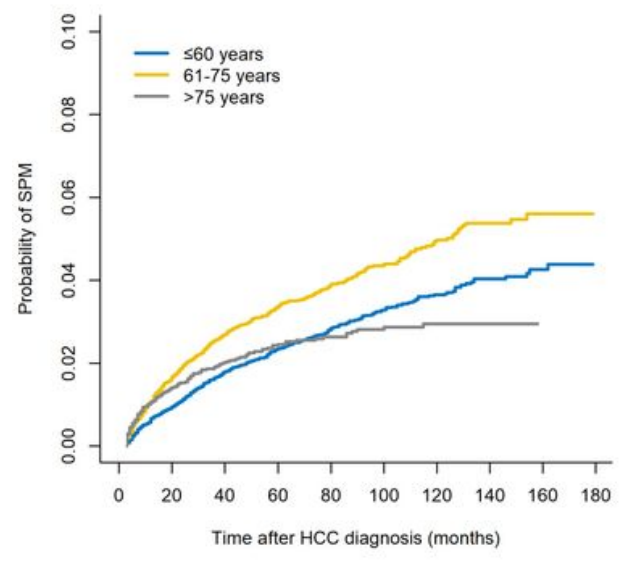

Marital status $(\mathrm{P}=0.002)$

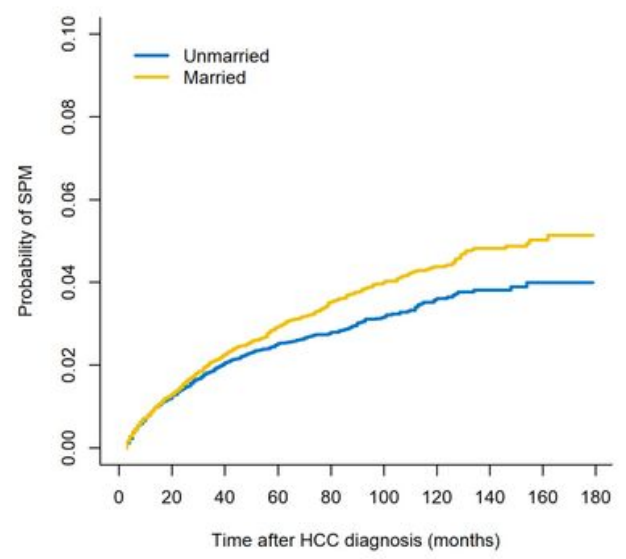

AJCC stage $(P<0.001)$

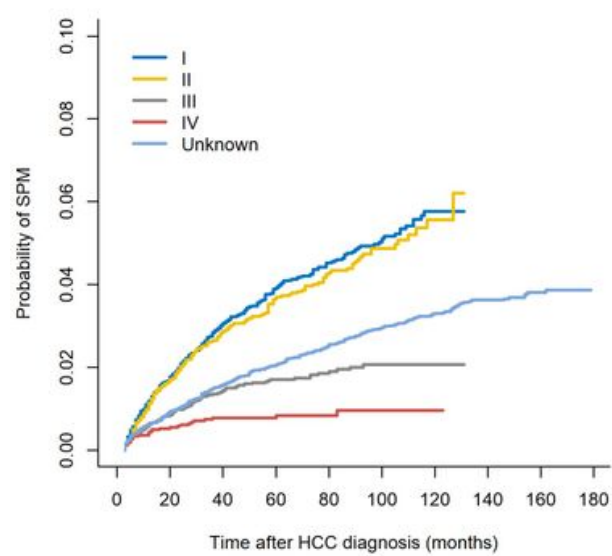

$\operatorname{Sex}(P=0.796)$

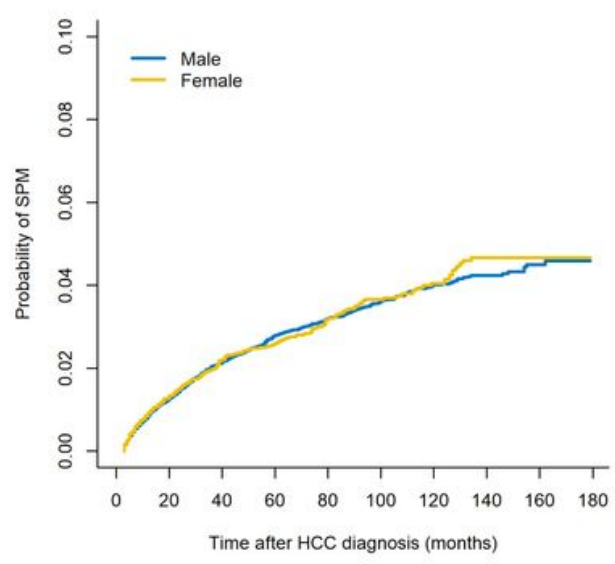

Tumor size $(\mathrm{P}<0.001)$

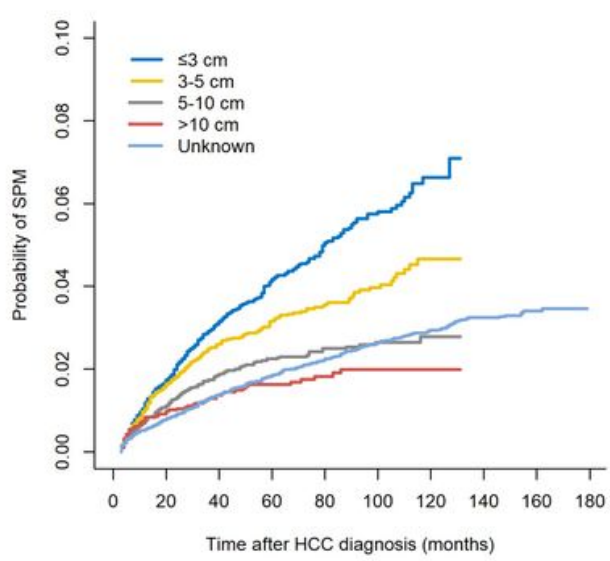

Surgery history $(\mathrm{P}<0.001)$

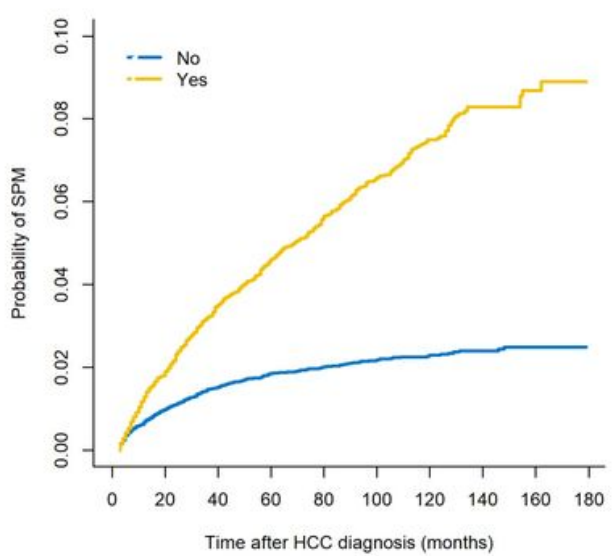

Race $(P=0.792)$

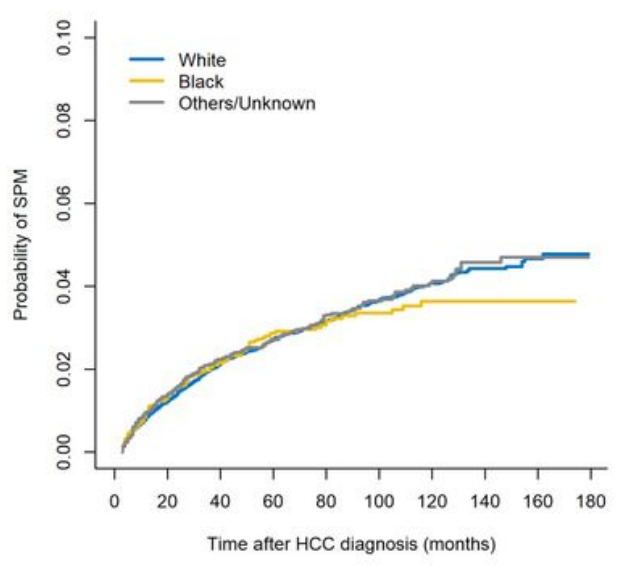

Histologic grade $(P<0.001)$

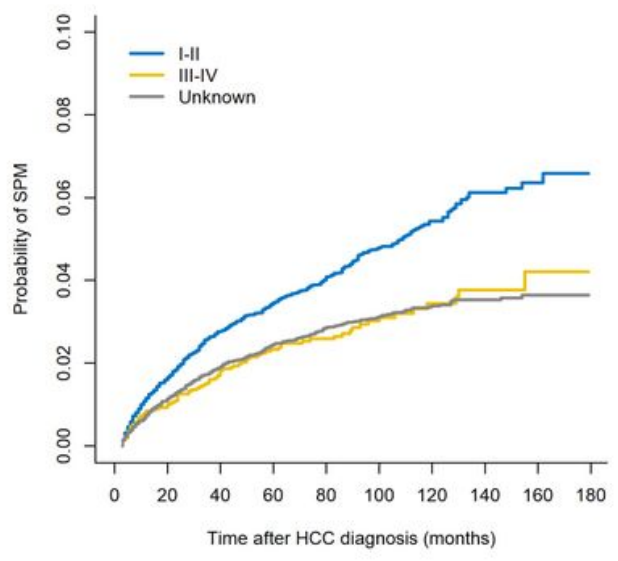

All

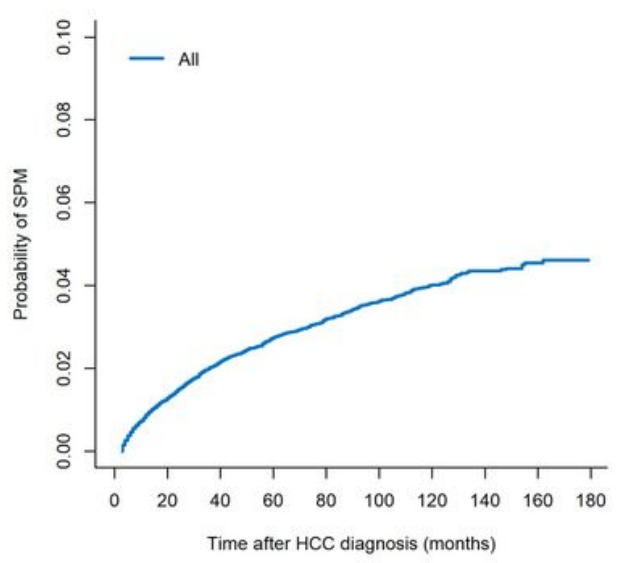

\section{Figure 1}

Curves for cumulative incidence of SPM after hepatocellular carcinoma based on each covariate. SPM, second primary malignancy. 


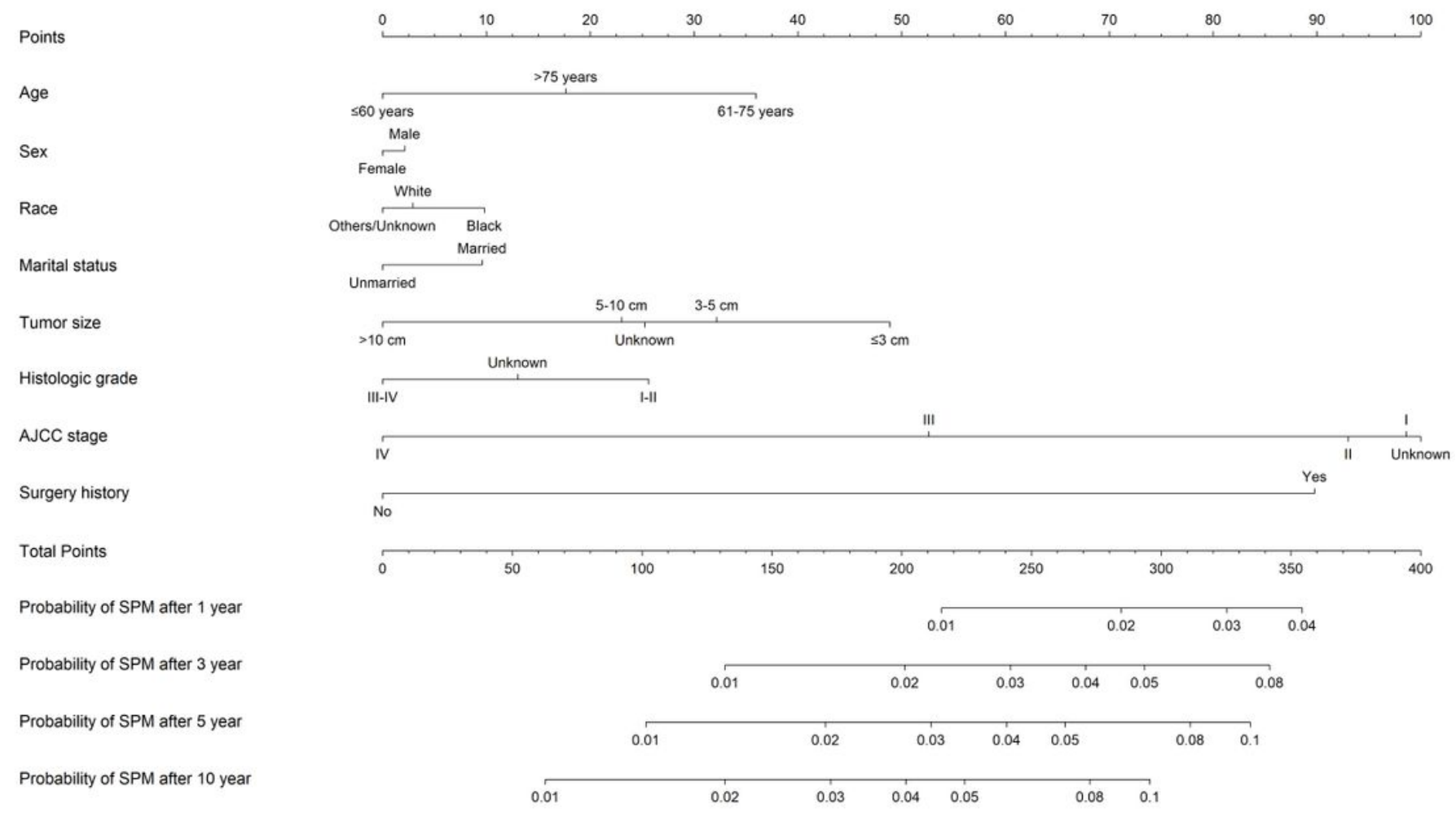

Figure 2

The competing-risk nomogram for the prediction of 1-, 3-, 5- and 10-year probabilities of SPM. SPM, second primary malignancy. 
A

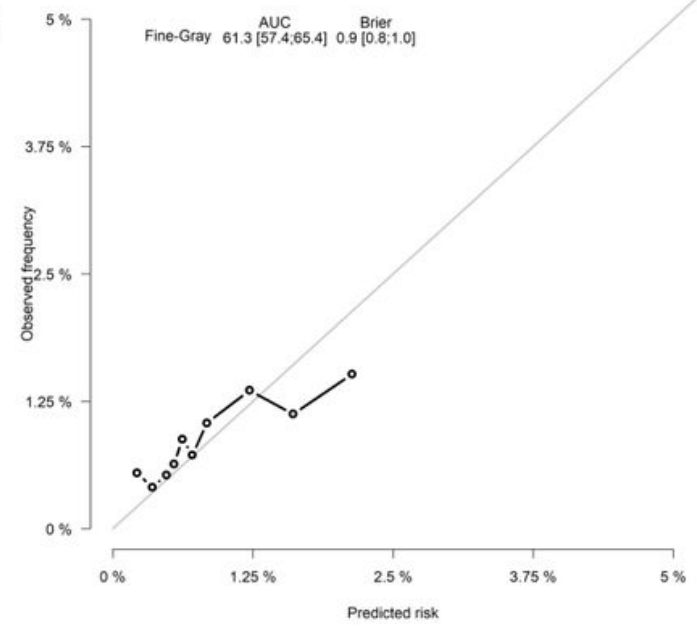

C

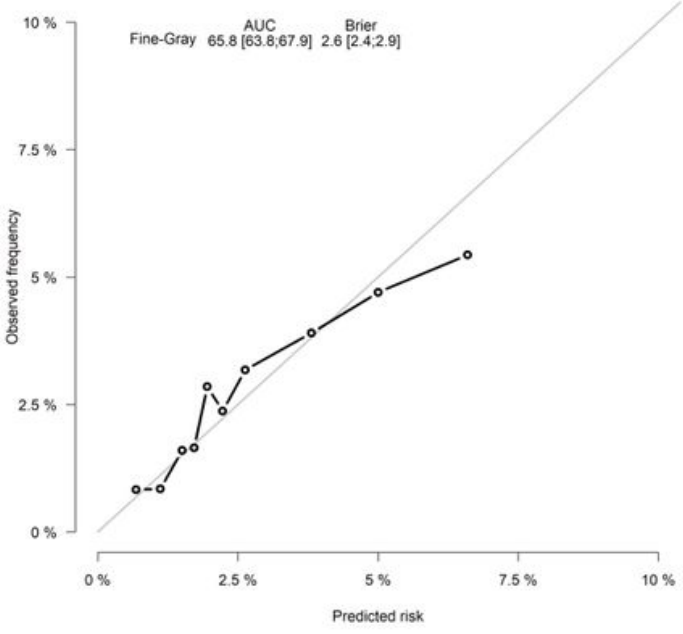

B

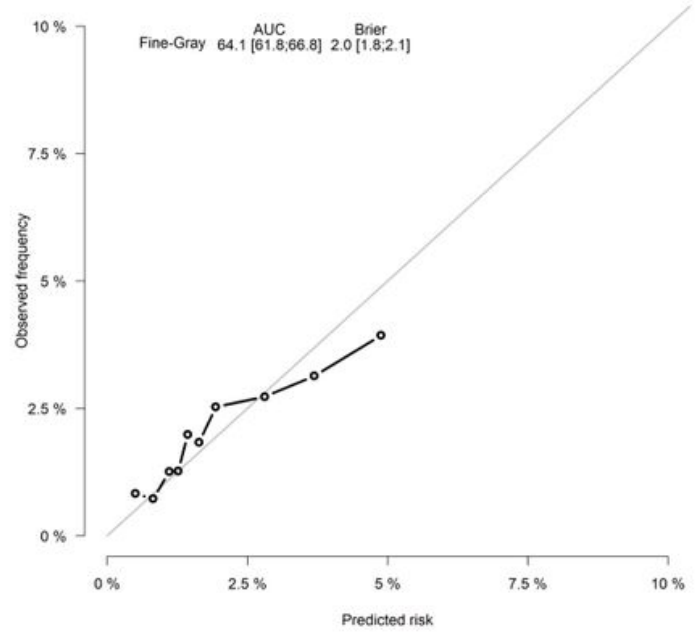

D

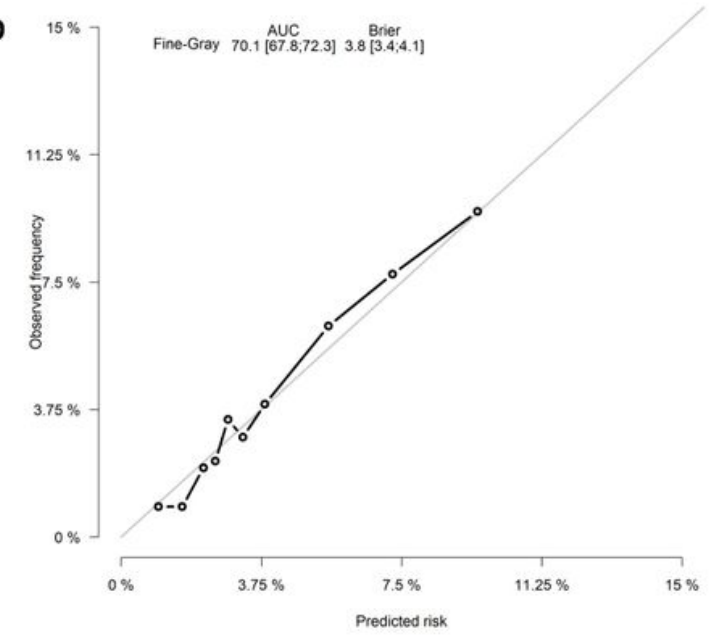

Figure 3

Calibration curves for the competing-risk nomogram in 1(A), 3(B), 5(C and 10(D) years. 


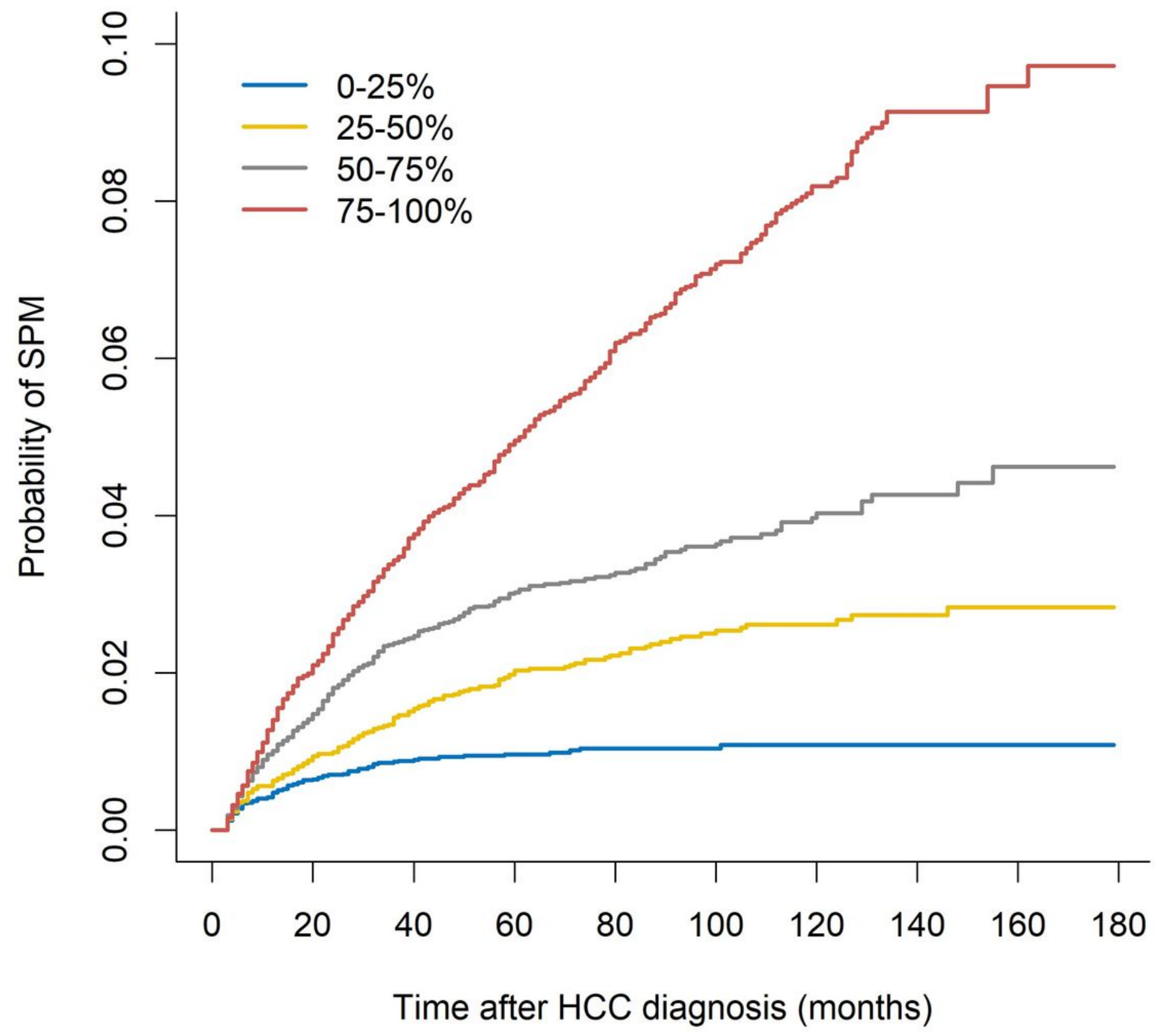

Figure 4

Validation for the classifying ability of predicting model. (A) Curves for cumulative incidence of SPM in each quartile; (B) The incidence of SPM in 10 years after HCC. SPM, second primary malignancy. 